manuscript No.

(will be inserted by the editor)

Friedrich Eisenbrand · Sören Laue

\title{
A Linear Algorithm for Integer Programming in the Plane
}

\begin{abstract}
We show that a 2-variable integer program, defined by $m$ constraints involving coefficients with at most $\varphi$ bits can be solved with $O(m+\varphi)$ arithmetic operations on rational numbers of size $O(\varphi)$.
\end{abstract}

\section{Introduction}

Integer programming is the problem of maximizing a linear function over the integer vectors which satisfy a given set of inequalities. A wide range of combinatorial optimization problems can be modeled as integer programming problems. But integer programming is not only related to combinatorics. The greatest common divisor of two numbers $a$ and $b \in \mathbb{Z}$ is the smallest integer combination $x a+y b$ such that $x a+y b \geqslant 1$. This is an integer program in two variables. This fact links integer programming also to the algorithmic theory of numbers.

The Euclidean algorithm requires $O(\varphi)$ arithmetic operations, if $\varphi$ is the binary encoding length of the input. Checking an integer point for feasibility, requires to test it for all the constraints. In this paper we prove that an integer program $\max \left\{c^{t} x \mid A x \leqslant b, x \in \mathbb{Z}^{2}\right\}$, where $c \in \mathbb{Z}^{2}, A \in \mathbb{Z}^{m \times 2}$ and $b \in \mathbb{Z}^{m}$ involve coefficients with at most $\varphi$ bits, can be solved with $O(m+\varphi)$ arithmetic operations on rationals of binary encoding length $O(\varphi)$. In the arithmetic complexity model, this is the best one can hope for if one believes that greatestcommon-divisor computation requires $\Omega(\varphi)$ arithmetic operations.

\section{Related work}

The two-variable integer programming problem has a long history. Polynomiality was established by Hirschberg and Wong [10] and Kannan [12] for special cases and by Scarf $[19,20]$ for the general case. Then, Lenstra [17] proved that integer programming in arbitrary fixed dimension can be solved in polynomial time.

Afterwards, various authors were looking for faster algorithms for the twodimensional case. Here is a table which summarizes the development of the last 20 years. In this table, $m$ denotes the number of constraints and $\varphi$ denotes the maximal binary encoding length of an involved coefficient.

Max-Planck-Institut für Informatik, Stuhlsatzenhausweg 85, 66123 Saarbrücken, Germany email: $\{$ eisen, soeren\}@mpi-sb.mpg.de 


\begin{tabular}{|c|c|}
\hline Method for integer programming & complexity \\
\hline \hline Feit [8], 1984 & $O(m \log m+m \varphi)$ \\
\hline Zamanskij and Cherkasskij [23], 1984 & $O(m \log m+m \varphi)$ \\
\hline Kanamaru, Nishizeki and Asano [11], 1994 & $O(m \log m+\varphi)$ \\
\hline Eisenbrand and Rote [7], 2001 & $O(m+(\log m) \varphi)$ \\
\hline Clarkson [4] combined with Eisenbrand [6] ${ }^{1}, 2003$ & $O(m+(\log m) \varphi)$ \\
\hline This paper & $O(m+\varphi)$ \\
\hline \hline Checking a point for feasibility & $\Theta(m)$ \\
\hline Greatest common divisor computation & $O(\varphi)$ \\
\hline
\end{tabular}

For comparison, we have also given the complexity of greatest-commondivisor computation and of checking whether a given integer point is feasible. Thus the last two lines of the table is the goal that one should aim for. This paper achieves this goal.

Our algorithm is the fastest algorithm in the arithmetic complexity model. Here, the basic arithmetic operations $+,-, *, /$ are unit-cost operations. This is in contrast to the bit-complexity model, where bit-operations are counted. In this model, the algorithm in [7] is the fastest known so far. Its complexity is $O(m+\log m \log \varphi) M(\varphi)$, where $M(\varphi)$ is the bit-complexity of $\varphi$-bit integer multiplication. In the bit-model, our algorithm can also be analyzed to require $O(m+\log m \log \varphi) M(\varphi)$ if the occurring shortest vector queries are individually carried out with Schönhage's algorithm [21].

It is well known, see, e.g. $[6,7,11]$ that, by means of an appropriate unimodular transformation, we can assume that the objective is to maximize the value of the first component. In fact, a reduction of a general integer programming problem to this special objective function requires one extended gcd-computation and a constant number of arithmetic operations. Thus we define the integer programming problem as follows.

Problem 1 (2IP). Given a system of inequalities $A x \leqslant b$, where $A \in \mathbb{Z}^{m \times 2}$ and $b \in \mathbb{Z}^{m}$, determine an integer point $x^{*} \in \mathbb{Z}^{2}$ which satisfies $A x \leqslant b$ and has maximal first component $x^{*}(1)$, or assert that $A x \leqslant b$ is integer infeasible.

In the following, the letter $m$ denotes the number of constraints of $A x \leqslant b$ and $\varphi$ is an upper bound on the binary encoding length of each constraint $a^{t} x \leqslant \beta$ of $A x \leqslant b$. We can also assume that the polyhedron $\left\{x \in \mathbb{R}^{2} \mid A x \leqslant b\right\}$ is bounded, thus that the constraints define a convex polygon $P=\left\{x \in \mathbb{R}^{2} \mid A x \leqslant b\right\}$.

\section{Preliminaries from algorithmic number theory}

In this section, we review some basics from algorithmic number theory, which are necessary to develop our algorithm.

1 This is a randomized method for arbitrary fixed dimension 


\subsection{The Euclidean algorithm and best approximations}

The Euclidean algorithm for computing the greatest common divisor $\operatorname{gcd}\left(a_{0}, a_{1}\right)$ of two integers $a_{0}, a_{1}>0$ computes the remainder sequence $a_{0}, a_{1}, \ldots, a_{k-1}, a_{k} \in$ $\mathbb{N}_{+}$, where $a_{i}, i \geqslant 2$ is given by $a_{i-2}=a_{i-1} q_{i-1}+a_{i}, q_{i} \in \mathbb{N}, 0<a_{i}<a_{i-1}$, and $a_{k}$ divides $a_{k-1}$ exactly. Then $a_{k}=\operatorname{gcd}\left(a_{0}, a_{1}\right)$. The extended Euclidean algorithm keeps track of the unimodular matrices $M^{(j)}=\prod_{i=1}^{j}\left(\begin{array}{cc}q_{i} & 1 \\ 1 & 0\end{array}\right), 0 \leqslant j \leqslant$ $k-1$. One has $\left(\begin{array}{c}a_{0} \\ a_{1}\end{array}\right)=M^{(j)}\left(\begin{array}{c}a_{j} \\ a_{j+1}\end{array}\right)$. The extended Euclidean algorithm requires $O(\varphi)$ arithmetic operations on $O(\varphi)$-bit integers, if the binary encoding length of $a_{0}$ and $a_{1}$ is $O(\varphi)$, see also $[15,1]$.

The fractions $M_{1,1}^{(i)} / M_{2,1}^{(i)}$ are called the convergents of $\alpha=a_{0} / a_{1}$. A fraction $x / y, y \geqslant 1$ is called a best approximation, ${ }^{2}$ if one has $|y \alpha-x|<\left|y^{\prime} \alpha-x\right|$ for all other fractions $x^{\prime} / y^{\prime}, 0<y^{\prime} \leqslant y$. A best approximation to $\alpha$ is a convergent of $\alpha$, see, e.g. [14].

\subsection{Lattices}

A 2-dimensional (rational) lattice $\Lambda$ is a set of the form $\Lambda(A)=\left\{A x \mid x \in \mathbb{Z}^{2}\right\}$, where $A \in \mathbb{Q}^{2 \times 2}$ is a nonsingular rational matrix. The matrix $A$ is called a basis of $\Lambda$. One has $\Lambda(A)=\Lambda(B)$ for $B \in \mathbb{Q}^{2 \times 2}$ if and only if $B=A U$ with some unimodular matrix $U$, i.e., $U \in \mathbb{Z}^{2 \times 2}$ and $\operatorname{det}(U)= \pm 1$. Every lattice $\Lambda(A)$ has a unique basis of the form $\left(\begin{array}{ll}a & b \\ 0 & c\end{array}\right) \in \mathbb{Q}^{2 \times 2}$, where $c>0$ and $a>b \geqslant 0$, called the Hermite normal form, $H N F$ of $\Lambda$, see, e.g. [22]. The Hermite normal form can be computed with an extended-gcd computation and a constant number of arithmetic operations.

A shortest vector of a lattice $\Lambda$ is a nonzero vector $v \in \Lambda-\{0\}$ with minimal $\ell_{\infty}$-norm $\|v\|_{\infty}=\max \{|v(i)| \mid i=1,2\}$. There are many algorithms known to compute a shortest vector of a 2 -dimensional lattice $[9,16,21]$. The following approach is very useful for our purposes.

Proposition $1\left([5]^{3}\right)$. Let $\Lambda \subseteq \mathbb{Q}^{2}$ be a rational lattice which is given by its Hermite normal form $\left(\begin{array}{ll}a & b \\ 0 & c\end{array}\right)$. A shortest vector of $\Lambda$ with respect to the $\ell_{\infty}$-norm is either $\left(\begin{array}{l}a \\ 0\end{array}\right)$ or $\left(\begin{array}{l}b \\ c\end{array}\right)$, or a vector of the form $\left(\begin{array}{c}-x a+y b \\ y c\end{array}\right)$, where the fraction $x / y$ is a best approximation of the number $b / a$.

Later, we will have to deal with the following problem for which we provide an algorithm below.

Problem 2. Given a lattice basis $A \in \mathbb{Q}^{2 \times 2}$ and a sequence of positive rational numbers $\alpha_{1}, \ldots, \alpha_{K}$, which are not all known in advance, but which reveal themselves one after the other. The task is to find a shortest vector w.r.t. the $\ell_{\infty}$-norm for each of the lattices $\Lambda_{i}$ generated by the matrices $\left(\begin{array}{ll}1 & 0 \\ 0 & \alpha_{i}\end{array}\right) \cdot A$, as $\alpha_{i}$ becomes available.

\footnotetext{
2 In [14] this is referred to as best approximation of the second kind

3 In [5] this assertion is stated for integral lattices. It is easy to see that it also holds for rational lattices
} 
Lemma 1. Let $A \in \mathbb{Q}^{2 \times 2}$ and $\alpha_{1}, \ldots, \alpha_{K}$ be parameters of Problem 2, where $A$ and each of the $\alpha_{i}$ have binary encoding length $O(\varphi)$. After a preprocessing step that involves $O(\varphi)$ arithmetic operations on rational numbers of size $O(\varphi)$, every shortest vector query can be answered in time $O(\log \varphi)$.

Proof. In the preprocessing step we first compute the Hermite normal form $\left(\begin{array}{ll}a & b \\ 0 & c\end{array}\right)$ of $A$ with the extended Euclidean algorithm. Then we compute all convergents $x_{j} / y_{j}, j=1, \ldots, k$ of $b / a$ with the extended Euclidean algorithm. From this, the convergents come out with the following property. The sequence $\left|-x_{j} a+y_{j} b\right|$ is monotonously decreasing and the sequence $y_{j} c$ is monotonously increasing and nonnegative. Since there are at most $O(\varphi)$ convergents of $b / a$, the preprocessing step takes time $O(\varphi)$.

For each shortest vector query we do the following. By Proposition 1 and since a best approximation of $b / a$ is a convergent of $b / a$, for each of the $\alpha_{i}$, we have to determine the convergent $x_{j} / y_{j}$ of $b / a$ such that $\left\|\left(\begin{array}{c}-x_{j} a+y_{j} b \\ y_{j} \alpha_{i} c\end{array}\right)\right\|_{\infty}$ is minimal. For this, we search the position $j_{i}$ in the list of convergents, where $\left|-x_{j_{i}} a+y_{j_{i}} b\right| \geqslant y_{j_{i}} \alpha_{i} c$ and $\left|-x_{j_{i}+1} a+y_{j_{i}+1} b\right|<y_{j_{i}+1} \alpha_{i} c$. If $\left|-x_{j} a+y_{j} b\right| \geqslant$ $y_{j} \alpha_{i} c$ holds for all convergents $x_{j} / y_{j}$, then $j_{i}$ shall be the second-last position. Similarly, if $\left|-x_{j} a+y_{j} b\right| \leqslant y_{j} \alpha_{i} c$ for all convergents $x_{j} / y_{j}$, then $j_{i}$ shall be the first position. The shortest vector of $\Lambda_{i}$ is then the shortest vector among the vectors

$$
\left(\begin{array}{c}
-x_{j_{i}} a+y_{j_{i}} b \\
y_{j_{i}} \alpha_{i} c
\end{array}\right),\left(\begin{array}{c}
-x_{j_{i}+1} a+y_{j_{i}+1} b \\
y_{j_{i}+1} \alpha_{i} c
\end{array}\right),\left(\begin{array}{c}
a \\
0
\end{array}\right),\left(\begin{array}{c}
b \\
\alpha_{i} c
\end{array}\right) .
$$

Since there are at most $O(\varphi)$ convergents of $b / a$, this position $j_{i}$, can be computed with binary search in $O(\log \varphi)$ steps. Thus a shortest vector query for a lattice $\Lambda_{i}$ generated by the matrix $\left(\begin{array}{ll}1 & 0 \\ 0 & \alpha_{i}\end{array}\right) \cdot A$ can be answered in time $O(\log \varphi)$.

\subsection{The flatness theorem}

A central concept of our algorithm, as in Lenstra's algorithm [17], is the lattice width of a convex body. Let $K \subseteq \mathbb{R}^{d}$ be a convex body. The width of $K$ along a direction $c \in \mathbb{R}^{d}$ is defined as $w_{c}(K)=\max \left\{c^{t} x \mid x \in K\right\}-\min \left\{c^{t} x \mid x \in\right.$ $K\}$. The lattice width $w(K)$ of a $K$ is defined as the minimum $w_{c}(K)$ over all nonzero vectors $c \in \mathbb{Z}^{d}-\{0\}$. Thus if a convex body has lattice width $\ell$ with a corresponding direction $c \in \mathbb{Z}^{d}$, then all its lattice points can be covered by at most $\lfloor\ell\rfloor+1$ parallel hyperplanes of the form $c^{t} x=\delta$, where $\delta \in \mathbb{Z} \cap\left[\min \left\{c^{t} x \mid\right.\right.$ $\left.x \in K\}, \max \left\{c^{t} x \mid x \in K\right\}\right]$. If a convex body does not contain any lattice points, then it must be thin in some direction, or equivalently its lattice width must be small. This is known as Khinchin's Flatness Theorem [13], see also [3].

Theorem 1 (Flatness theorem). There exists a constant $f(d)$ depending only on the dimension $d$, such that each full-dimensional convex and compact body $K \subseteq \mathbb{R}^{d}$ containing no integer point has width less than $f(d)$. 
It is not difficult to see that $f(d)$ can be chosen to be any number larger than $d^{n / 5}$, see, e.g. [3]. Thus $f(2)=2.9$ satisfies our needs. One can show [2] that $f(d)=O\left(d^{3 / 2}\right)$.

How can the width of a convex body be computed? In this paper, we only need to do this for triangles. Let $T=\operatorname{conv}(u, v, w) \subseteq \mathbb{R}^{2}$ be a triangle. The width is invariant under translation. Thus the width of $T$ is the width of the triangle $T^{\prime}=\operatorname{conv}(0, v-u, w-u)$. The width of $T^{\prime}$ along a vector $c \in \mathbb{R}^{2}$ is then by defintion

$$
\begin{aligned}
w_{c}\left(T^{\prime}\right) & =\max \left\{0, c^{t}(v-u), c^{t}(w-u)\right\}-\min \left\{0, c^{t}(v-u), c^{t}(w-u)\right\} \\
& =\max \left\{0, c^{t}(v-u), c^{t}(w-u)\right\}+\max \left\{0,-c^{t}(v-u),-c^{t}(w-u)\right\} .
\end{aligned}
$$

Obviously, it is bounded from below by $\max \left\{\left|c^{t}(v-u)\right|,\left|c^{t}(w-u)\right|\right\}$ and bounded from above by $2 \max \left\{\left|c^{t}(v-u)\right|,\left|c^{t}(w-u)\right|\right\}$. Let $A_{T} \in \mathbb{R}^{2}$ be the matrix $A_{T}=$ $\left(\begin{array}{c}(v-u)^{t} \\ (w-u)^{t}\end{array}\right)$. The width along $c$ thus satisfies the following relation

$$
\left\|A_{T} c\right\|_{\infty} \leqslant w_{c}(T) \leqslant 2\left\|A_{T} c\right\|_{\infty} .
$$

This means that the width of $T$ is bounded from below by the length of the shortest (infinity norm) vector of $\Lambda\left(A_{T}\right)$ and bounded from above by twice the length of the shortest vector of $\Lambda\left(A_{T}\right)$. Furthermore, if $v=A_{T} c$ is a shortest vector, then the following relation holds

$$
w_{c}(T) \leqslant w(T) \leqslant 2 w_{c}(T) .
$$

In the sequel, we call a vector $c \in \mathbb{Z}^{2}$, such that $v=A_{T} c$ is a shortest vector of $\Lambda\left(A_{T}\right)$, a thin direction of $T$. A shortest vector of $\Lambda\left(A_{T}\right)$ w.r.t. the $\ell_{\infty}$-norm will be denoted as a shortest vector of the triangle $T$. Its length is denoted by $\mathrm{SV}(T)$.

\section{Partitioning the polygon}

In a first step, we partition the polygon into four parts. Two of the parts belong to a class of polygons for which one already knows an $O(m+\varphi)$ algorithm for their corresponding integer programs [7]. In the following sections, we will deal with the other two polygons.

First we compute the rightmost point and the leftmost point of $P$ and we consider the line $g$ through these two points, see Figure 1 . This line dissects $P$ into an upper part $P_{U}$ and a lower part $P_{L}$. Next we compute vertices of $P_{U}$ and $P_{L}$ which have largest distance from the line $g$ and draw a vertical line $h_{U}$ and $h_{L}$ through these points. The line $h_{U}$ dissects $P_{U}$ again in two parts, an upper-left polygon $P_{U l}$ and an upper-right polygon $P_{U r}$. The line $h_{L}$ partitions $P_{L}$ into two parts, a lower-left polygon $P_{L l}$ and a lower-right polygon $P_{L r}$. The optimum integer point in $P$ is the maximum of the optima of these four polygons. This partition can be found with linear programming. Using the algorithm of Megiddo [18], this requires $O(m)$ operations. Notice that the binary encoding length of each constraint describing the four polygons remains $O(\varphi)$. 


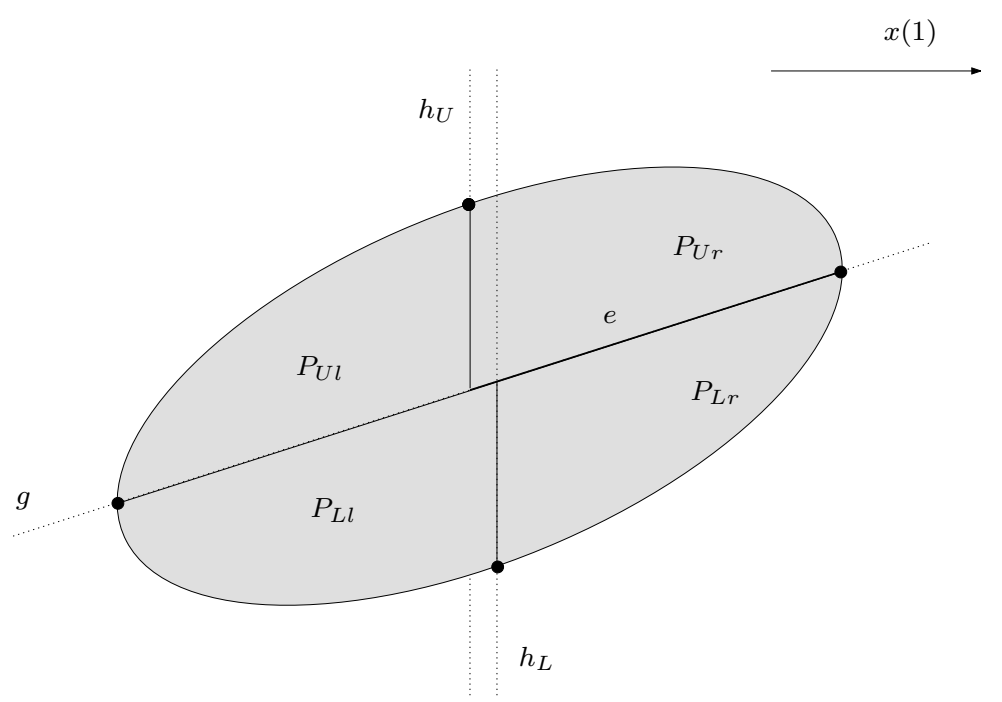

Figure 1: The dissection of the polygon $P$. The arrow is the $x(1)$-direction in which we optimize.

The polygons $P_{U l}$ and $P_{L l}$ are lower polygons in the terminology of Eisenbrand and Rote [7]. This is because the set of points with the largest objective value form an edge that is parallel to the objective line and there are two parallel lines trough the endpoints of this edge which enclose the polygon. ${ }^{4}$ Thus Proposition 1 and Theorem 2 of [7] implies that the integer program over $P_{U l}$ and $P_{L l}$ can be solved in with $O(m+\varphi)$ arithmetic operations on rationals with $O(\varphi)$ bits. For the sake of completeness, we sketch this algorithm in the appendix.

The polygons $P_{U r}$ and $P_{L r}$ are the ones we need to take care of. The polygon $P_{U r}$ has a special structure. It has an edge $e$, such that each point of $P_{U r}$ lies vertically above $e$ and the two vertical lines through the endpoints of this edge enclose the polygon. Furthermore, the vertical line through the vertex on the left of $e$, defines a facet of $P_{U r}$. All the other facets, from left to right, have decreasing slope and each slope is at most the slope of $e$. A polygon of this kind will be called a polygon of upper-right kind in the sequel. Notice that $P_{L r}$ becomes an upper-right polygon, when it is reflected around the $x(1)$ axis. Therefore we concentrate now on the solution of integer programming problems over polygons of upper-right kind.

\footnotetext{
${ }^{4}$ In [7] the objective is to find a highest integer point, while we find a rightmost integer point
} 


\section{A prune-and-search algorithm}

In the following, let $P$ be a polygon of upper-right kind. We now present an $O(m+\varphi)$ algorithm for this case. Similar to the algorithm in [7] we use the prune-and-search technique of Megiddo [18] to solve the optimization problem over $P$.

The idea is to search for a parameter $\ell$, such that the truncated polygon $P_{\ell}=$ $P \cap(x(1) \geqslant \ell)$ has width $w\left(P_{\ell}\right)$ between $f(2)$ and $4 f(2)$. If we have found such an $\ell$, we know two important things. First, the flatness theorem guarantees that $P_{\ell}$ is feasible and thus that the optimum of the integer programming problem over $P$ lies in $P_{\ell}$. Furthermore, all lattice points of $P_{\ell}$, an therefore also the optimum, must lie on at most $4 f(2)+1$ parallel line segments in the corresponding flat direction. Thus, we have reduced the integer programming problem over $P$ to the problem of finding an optimum of a constant number of one-dimensional integer programming problems, which then can be solved in $O(\varphi)$ steps each.

We will approximate the width of $P_{\ell}$ as follows. Consider the edge $f$ of $P_{\ell}$ induced by the constraint $x(1) \geqslant \ell$ and the edge $e^{\prime}$, which emerges from the lower edge $e$ of $P$ intersected with $(x(1) \geqslant \ell)$. The convex hull of both edges is a triangle $T_{\ell}$, see, Figure 2.

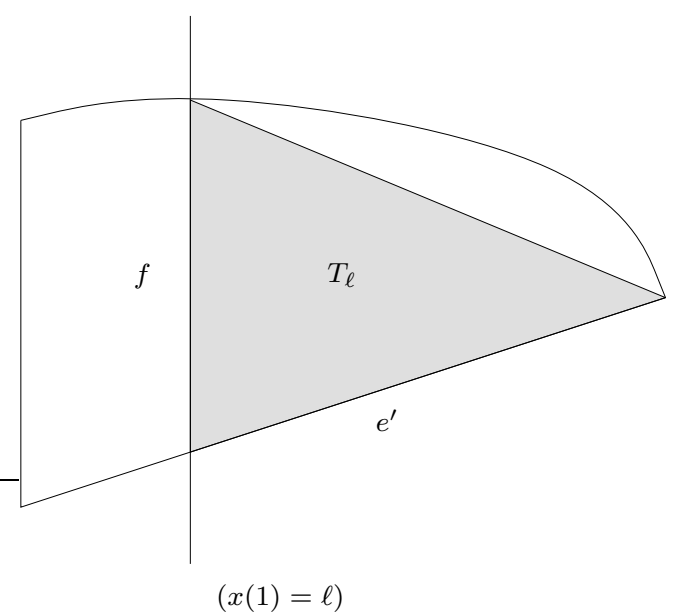

Figure 2: The polygon $P_{\ell}$ and the triangle $T_{\ell}$.

Obviously, we have $T_{\ell} \subseteq P_{\ell}$. It is easy to see that if we scale $T_{\ell}$ by a factor of 2 and translate $T_{\ell}$ appropriately, then it includes $P_{\ell}$. Hence, the width $w\left(P_{\ell}\right)$ satisfies $w\left(T_{\ell}\right) \leqslant w\left(P_{\ell}\right) \leqslant 2 w\left(T_{\ell}\right)$. From Section 2.3 we can conclude $\mathrm{SV}\left(T_{\ell}\right) \leqslant$ $w\left(P_{\ell}\right) \leqslant 4 \mathrm{SV}\left(T_{\ell}\right)$. Thus, we are interested in a parameter $\ell$, such that the shortest vector of $T_{\ell}$ has length $f(2)$.

We start with $m$ constraints and maintain two numbers $\ell_{\text {thick }}$ and $\ell_{\text {thin }}$. In the beginning, $\ell_{\text {thick }}$ is the $x(1)$-component of the left endpoint of the edge $e$ and $\ell_{\text {thin }}$ 
is the $x(1)$-component of the right endpoint of the edge $e$. If $\mathrm{SV}\left(T_{\ell_{t h i c k}}\right) \leqslant f(2)$, then $P$ itself is flat an we are done. Otherwise we keep the following invariant.

The shortest vector of $T_{\ell_{\text {thick }}}$ has length at least $f(2)$ and the shortest vector of $T_{\ell_{\text {thin }}}$ has length at most $f(2)$.

The idea is to prune constraints, while we search for the correct position $\ell$, which cannot be facet defining for the intermediate part of the polygon $P \cap$ $\left(x(1) \geqslant \ell_{\text {thick }}\right) \cap\left(x(1) \leqslant \ell_{\text {thin }}\right)$, see Figure 3.

One iteration is as follows. We pair up all $m$ constraints yielding $m / 2$ intersection points. Then we compute the x-median $\ell_{\text {med }}$ of the intersection points. Now we distinguish three cases. One is that $\ell_{\text {med }}$ lies to the right of $\ell_{\text {thin }}$. In this case, we can delete from each pair of intersection points to the right of the median, the constraint with the smaller slope. We can do this, since this constraint cannot be facet-defining for the intermediate polygon. Similarly, if $\ell_{\text {med }}$ lies to the left of $\ell_{\text {thick }}$, we can delete from each pair on the left of the median the constraint with the larger slope.

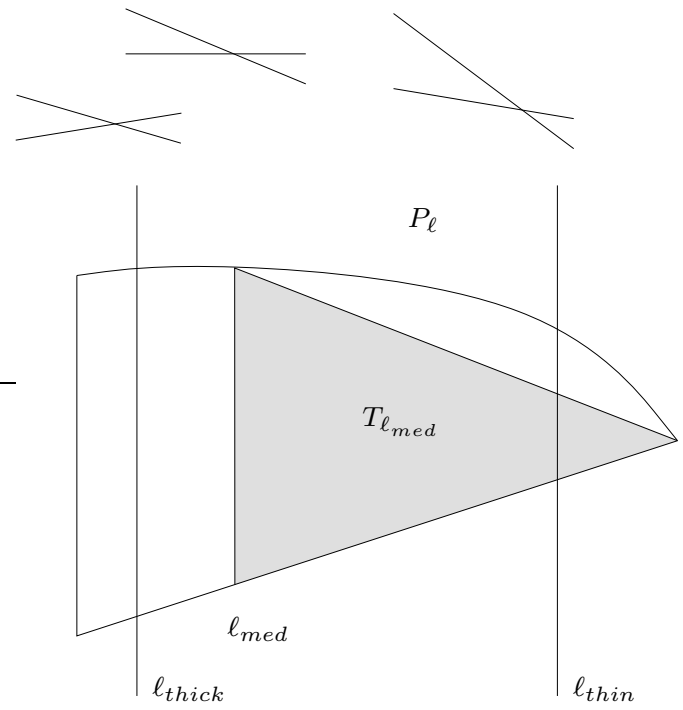

Figure 3: The prune-and-search algorithm for a polygon of the upper-right kind

The more interesting case is the one, where $\ell_{\text {med }}$ lies in-between $\ell_{\text {thick }}$ and $\ell_{\text {thin }}$. Then we compute the length of the edge $f$ which is induced by $x(1) \geqslant \ell_{\text {med }}$. This edge is simply the line-segment spanned by the intersection of $x(1)=\ell_{\text {med }}$ with $e$ and by the lowest intersection point of the line $x(1)=\ell_{m e d}$ with all $m$ constraints.

Now we compute the shortest vector of $T_{\ell_{m e d}}$. If its length is smaller than $f(2)$, then we set $\ell_{\text {thin }}$ to $\ell_{m e d}$ and delete from each intersection point that lies 
to the right of $\ell_{\text {med }}$ the constraint with the smaller slope. Otherwise we set $\ell_{\text {thick }}$ to $\ell_{m e d}$ and delete from each intersection point that lies to the left of $\ell_{m e d}$ the constraint with the larger slope.

We repeat this prune and search procedure until we have found a position $\ell$, where the shortest vector of $T_{\ell}$ is $f(2)$ or we identified a constant number of constraints, which can be facet defining for $P \cap\left(x(1) \geqslant \ell_{\text {thick }}\right) \cap\left(x(1) \leqslant \ell_{\text {thin }}\right)$.

In the first case, we know that the optimum lies in $P_{\ell}$ and we have a flat direction of $P_{\ell}$, namely the vector $c \in \mathbb{Z}^{2}-\{0\}$ such that $v=A_{T_{\ell}} c$ is a shortest vector of $T_{\ell}$. Thus the optimum is the largest of the optima of the integer programs over the constant number line segments $P_{\ell} \cap\left(c^{t} x=\delta\right)$, where $\delta \in \mathbb{Z} \cap\left[\min \left\{c^{t} x \mid x \in P_{\ell}\right\}, \max \left\{c^{t} x \mid x \in P_{\ell}\right\}\right]$. In the second case, we know that the optimum lies in $P_{\ell_{\text {thick }}}$. Furthermore, $P_{\ell_{\text {thick }}}$ can be partitioned into $P_{\ell_{\text {thin }}}$, and the polygon $P \cap\left(x(1) \geqslant \ell_{\text {thick }}\right) \cap\left(x(1) \leqslant \ell_{t h i n}\right)$. The first polygon is flat. The second polygon is defined by a constant number of constraints, for which integer programming can be solved with $O(\varphi)$ arithmetic operations.

\section{Analysis}

We will prove that the presented algorithm runs in $O(m+\varphi)$ using rational numbers of size $O(\varphi)$.

Suppose we are in the $i$-th round of the prune-and-search algorithm and suppose we are left with $m_{i}$ constraints. In this round we compute $m_{i} / 2$ intersection points, the median of them, the corresponding triangle $T_{\ell}$ and query for the shortest vector of $T_{\ell}$. Hence, the running time for round $i$, without considering the shortest vector queries, is $O\left(m_{i}\right)$. We discard $1 / 4$ of the constraints. Therefore, the overall running time of the prune-and-search algorithm without considering the shortest vector queries is $O(m)$.

Let us consider the shortest vector queries. Let $T$ be the first triangle, for which we compute the shortest vector. The angle, which is enclosed by the edges $f$ and $e^{\prime}$ is the same for all triangles for which we query a shortest vector. Let $A_{T}$ be the matrix of $T$ as it is defined at the end of Section 2.3. The matrices $A_{T_{\ell}}$ of the following triangles thus satisfy

$$
A_{T_{\ell}}=\beta_{\ell} \cdot\left(\begin{array}{cc}
1 & 0 \\
0 & \alpha_{\ell}
\end{array}\right) \cdot A_{T},
$$

with rational numbers $\alpha_{\ell}$ and $\beta_{\ell}$ which can be computed from $T$ and $T_{\ell}$ in constant time. The length of the shortest vector of $T_{\ell}$ is equal to $\beta_{\ell}$ times the length of the shortest vector of the lattice $\Lambda\left(\left(\begin{array}{cc}1 & 0 \\ 0 & \alpha_{\ell}\end{array}\right) \cdot A_{T}\right)$. Hence, we can apply Lemma 1. As we perform $O(\log m)$ queries, the total number shortest vector queries can be computed with $O(\varphi+\log m \cdot \log \varphi)$ arithmetic operations on rational numbers of size $O(\varphi)$. Thus the total running time amounts to $O(m+$ $\varphi+\log m \cdot \log \varphi)=O(m+\varphi)$ arithmetic operations on rational numbers of binary encoding length $O(\varphi)$, which proves our main result.

Theorem 2. A two-variable integer programming problem $\max \left\{c^{t} x \mid A x \leqslant\right.$ $\left.b, x \in \mathbb{Z}^{2}\right\}$, where $A \in \mathbb{Z}^{m \times 2}$ and $b \in \mathbb{Z}^{m}$ and $c \in \mathbb{Z}^{2}$ involve only coefficients of 
binary encoding length $O(\varphi)$, can be solved with $O(\varphi+m)$ arithmetic operations on rational numbers of size $O(\varphi)$.

\section{Appendix}

For the sake of completeness, we shortly sketch how to optimize over a polygon of the upper-left kind. A more elaborated analysis can be found in [7].

Suppose, we are given a polygon $P$ of the upper-left kind as in Figure 4. Similarly to the algorithm for a polygon of the upper-right kind, we search for a parameter $\ell$, such that the truncated polygon $P_{\ell}=P \cap(x(1) \geqslant \ell)$ has width $w\left(P_{\ell}\right)$ between $f(2)$ and $4 f(2)$. We then know, that $P_{\ell}$ contains the optimal integer point and all integer points can be covered by at most $4 f(2)$ parallel line segments.

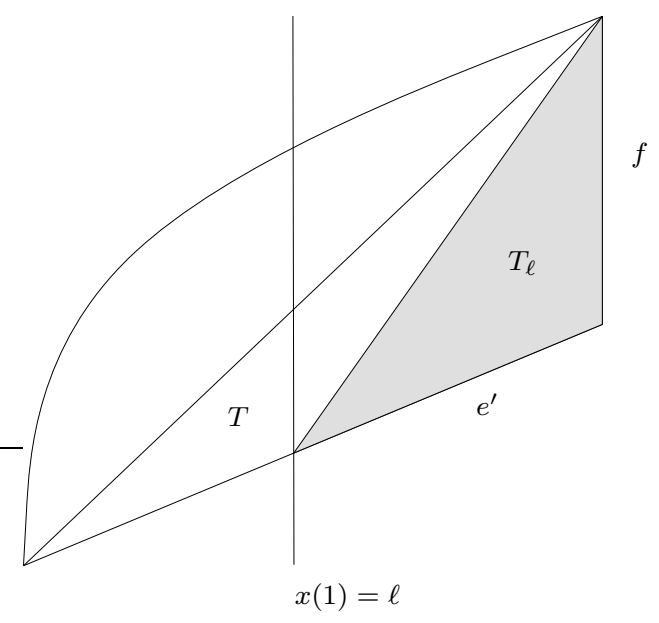

Figure 4: A polygon of the upper-left kind

Analogously to Section 4 , we approximate the polygon $P_{\ell}$ by a triangle $T_{\ell}$. Obviously $T_{\ell} \subseteq P_{\ell}$ holds. It is easy to see that if we scale $T_{\ell}$ by factor of 2 , it then includes $P_{\ell}$. Hence, the length of a shortest vector of $T_{\ell}$ is a lower bound for the width of $P_{\ell}$ and four times the length of a shortest vector of $T_{\ell}$ is an upper bound for the width of $P_{\ell}$.

The triangles $T_{\ell}$ are the convex hull of their edges $f$ and $e^{\prime}$, see Figure 4 . Obviously, the edge $f$ is the same for all triangles $T_{\ell}$. Only the $e^{\prime}$ differ, they are scaled copies of each other.

Let $T$ be the triangle in $P$ as indicated in Figure 4 and let $A_{T}$ be its matrix as defined in Section 2.3. Then, the matrices of all triangles $T_{\ell}$ are of the form

$$
A_{T_{\ell}}=\left(\begin{array}{cc}
1 & 0 \\
0 & \alpha_{\ell}
\end{array}\right) \cdot A_{T} .
$$


Hence, we only need to look for a rational number $\alpha$, such that the corresponding shortest vector of $A_{T_{\ell}}$ has length $f(2)$. Applying Proposition 1 and the Euclidean algorithm we can do this using $O(\varphi)$ arithmetic operations on rational numbers of length $O(\varphi)$ as follows. Compute the convergents $x_{j} / y_{j}$ of the $b / a$, where $\left(\begin{array}{ll}a & b \\ 0 & c\end{array}\right)$ is the Hermite normal form of $A_{T}$. We then search the first position $j$ in the list of convergents, where $\left|-x_{j} a+y_{j} b\right|$ is at most $f(2)$. The rational number $\alpha$ is then the solution to the equation $\alpha y_{j} c=f(2)$.

If we have found the parameter $\ell$, we can reduce the original problem to finding the optimal integer point of a constant number of one-dimensional integer problems which can be solved in $O(\varphi)$ steps each.

\section{Acknowledgements}

We would like to thank an anonymous referee of this journal and a referee of ISAAC for helpful remarks and suggestions.

\section{References}

1. A. V. Aho, J. E. Hopcroft, and J. D. Ullman. The Design and Analysis of Computer Algorithms. Addison-Wesley, Reading, 1974.

2. W. Banaszczyk, A. E. Litvak, A. Pajor, and S. J. Szarek. The flatness theorem for nonsymmetric convex bodies via the local theory of Banach spaces. Mathematics of Operations Research, 24(3):728-750, 1999.

3. A. Barvinok. A course in convexity, volume 54 of Graduate Studies in Mathematics. American Mathematical Society, Providence, RI, 2002.

4. K. L. Clarkson. Las Vegas algorithms for linear and integer programming when the dimension is small. Journal of the Association for Computing Machinery, 42:488-499, 1995.

5. F. Eisenbrand. Short vectors of planar lattices via continued fractions. Information Processing Letters, 79(3):121-126, 2001.

6. F. Eisenbrand. Fast integer programming in fixed dimension. In G. D. Battista and U. Zwick, editors, In Proceedings of the 11th Annual European Symposium on Algorithms, ESA' 2003, volume 2832 of LNCS, pages 196-207. Springer, 2003. to appear in Computing.

7. F. Eisenbrand and G. Rote. Fast 2-variable integer programming. In K. Aardal and B. Gerards, editors, Integer Programming and Combinatorial Optimization, IPCO 2001 , volume 2081 of $L N C S$, pages 78-89. Springer, 2001.

8. S. D. Feit. A fast algorithm for the two-variable integer programming problem. Journal of the Association for Computing Machinery, 31(1):99-113, 1984.

9. C. F. Gauß. Disquisitiones arithmeticae. Gerh. Fleischer Iun., 1801.

10. D. S. Hirschberg and C. K. Wong. A polynomial algorithm for the knapsack problem in two variables. Journal of the Association for Computing Machinery, 23(1):147-154, 1976.

11. N. Kanamaru, T. Nishizeki, and T. Asano. Efficient enumeration of grid points in a convex polygon and its application to integer programming. International Journal of Computational Geometry \& Applications, 4(1):69-85, 1994.

12. R. Kannan. A polynomial algorithm for the two-variable integer programming problem. Journal of the Association for Computing Machinery, 27(1):118-122, 1980.

13. R. Kannan and L. Lovász. Covering minima and lattice-point-free convex bodies. Annals of Mathematics, 128:577-602, 1988.

14. A. Y. Khintchine. Continued Fractions. Noordhoff, Groningen, 1963.

15. D. Knuth. The art of computer programming, volume 2. Addison-Wesley, 1969.

16. J. C. Lagarias. Worst-case complexity bounds for algorithms in the theory of integral quadratic forms. Journal of Algorithms, 1:142-186, 1980.

17. H. W. Lenstra. Integer programming with a fixed number of variables. Mathematics of Operations Research, 8(4):538 - 548, 1983. 
18. N. Megiddo. Linear programming in linear time when the dimension is fixed. Journal of the Association for Computing Machinery, 31:114-127, 1984.

19. H. E. Scarf. Production sets with indivisibilities. Part I: generalities. Econometrica, 49:1$32,1981$.

20. H. E. Scarf. Production sets with indivisibilities. Part II: The case of two activities. Econometrica, 49:395-423, 1981.

21. A. Schönhage. Fast reduction and composition of binary quadratic forms. In International Symposium on Symbolic and Algebraic Computation, ISSAC 91, pages 128-133. ACM Press, 1991.

22. A. Schrijver. Theory of Linear and Integer Programming. John Wiley, 1986.

23. L. Y. Zamanskij and V. D. Cherkasskij. A formula for determining the number of integral points on a straight line and its application. Ehkon. Mat. Metody, 20:1132-1138, 1984. (in Russian). 